\title{
„Deutscher Qualitätspreis Gesundheit 2009“ an Prof. Hans Konrad Selbmann
}

\section{Zusammenfassung}

Im Rahmen des „3. Nationalen Qualitätskongresses Gesundheit“ erhielt Prof. Dr. Hans Konrad Selbmann am 26. November in Berlin den mit 10.000 Euro dotierten „Deutschen Qualitätspreis Gesundheit 2009“. Mit der Auszeichnung würdigt "Gesundheitsstadt Berlin" Selbmanns vorbildliches Engagement auf dem Gebiet der Qualitätssicherung und Patientensicherheit in der Gesundheitsversorgung.

\section{Text}

Selbmann sei der „Wegbereiter für die Qualitätssicherung in der Medizin in Deutschland“, betonte der Parlamentarische Staatssekretär im Bundesgesundheitsministerium, Daniel Bahr (FDP), in seiner Rede zur Übergabe des Preises.

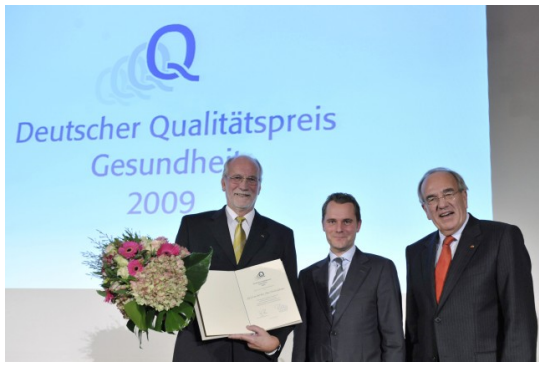

Bildquelle: obs/Gesundheitsstadt Berlin

Prof. Dr. Hans Konrad Selbmann leitete bis 2007 das Institut für medizinische Informationsverarbeitung der Universität Tübingen. Zu Selbmanns großen Verdiensten gehören u. a. die Begleitung der bayerischen Perinatalerhebung ab 1978 und die Gründung der „Gesellschaft für Qualitätsmanagement in der Gesundheitsversorgung“ 1993. Das Preisgeld in Höhe von 10.000 Euro will Prof. Selbmann der Arbeitsgemeinschaft der Wissenschaftlichen Medizinischen Fachgesellschaften (AWMF) für ihr neu gegründetes "AWMF-Institut für Medizinisches Wissensmanagement" in Marburg zur Verfügung stellen, um die Vernetzung von Leitlinien mit Qualitätsmanagement und von Leitlinienentwicklern mit Qualitätsmanagern weiter voranzutreiben.

Zu dieser Vernetzung tragen nach Angaben von Prof. Selbmann z.B. bei:

- die Ableitung von Qualitätsindikatoren aus hochwertigen Leitlinien,

- die Einbringung von Leitlinien in die Zertifizierungsprozesse von Einrichtungen,

\author{
Wolfgang Müller
}

1 AWMF, Düsseldorf
- das Benchmarking zwischen Leistungserbringern mit Hilfe der Qualitätsindikatoren und der dahinterstehenden Leitlinie,

- die Entwicklung und Erprobung von Methoden der lokalen Adaptierung von Leitlinien,

- Verfahren zur Umsetzung von Leitlinien im Versorgungsalltag,

- die Schaffung von Patientenfassungen von Leitlinien, um Patienten stärker an der Anwendung von Leitlinien zu beteiligen.

Die AWMF gratuliert ihrem Präsidiumsmitglied Prof. Selbmann herzlich zu dieser Auszeichnung und dankt inm besonders für die in Aussicht gestellte Spende des Preisgeldes zugunsten des AWMF-Instituts für medizinisches Wissensmanagement in Marburg (Leitung: Frau Prof. Dr. Ina Kopp), das sich zur Zeit im Aufbau befindet.

\section{Korrespondenzadresse:}

M.A. Wolfgang Müller

AWMF-Geschäftsstelle, Ubierstr. 20, 40223 Düsseldorf office@awmf.org

\section{Bitte zitieren als}

Müller W. „Deutscher Qualitätspreis Gesundheit 2009“ an Prof. Hans Konrad Selbmann. GMS Mitt AWMF. 2009;6:Doc24.

\section{Artikel online frei zugänglich unter}

http://www.egms.de/en/journals/awmf/2009-6/awmf000197.shtml

\section{Eingereicht: 07.12.2009}

Veröffentlicht: 14.12.2009

\section{Copyright}

C2009 Müller. Dieser Artikel ist ein Open Access-Artikel und steht unter den Creative Commons Lizenzbedingungen

(http://creativecommons.org/licenses/by-nc-nd/3.0/deed.de). Er darf vervielfältigt, verbreitet und öffentlich zugänglich gemacht werden, vorausgesetzt dass Autor und Quelle genannt werden. 